Discrete Comput Geom 34:381-390 (2005)

DOI: $10.1007 / \mathrm{s} 00454-005-1183-1$

\title{
Transversals to Line Segments in Three-Dimensional Space*
}

\author{
H. Brönnimann, ${ }^{1}$ H. Everett,${ }^{2}$ S. Lazard, ${ }^{2}$ F. Sottile, ${ }^{3}$ and S. Whitesides ${ }^{4}$ \\ ${ }^{1}$ Department of Computer and Information Science, Polytechnic University, \\ Brooklyn, NY 11201, USA \\ hbr@poly.edu \\ ${ }^{2}$ LORIA Inria-Lorraine et Université Nancy 2, \\ 54506 Vandœuvre-lès-Nancy Cedex, France \\ \{everett,lazard\}@loria.fr \\ ${ }^{3}$ Department of Mathematics, Texas A\&M University, \\ College Station, TX 77843-3368, USA \\ sottile@math.tamu.edu \\ ${ }^{4}$ School of Computer Science, McGill University, \\ Montréal, Quebec, Canada H3A 2A7 \\ sue@cs.mcgill.ca
}

\begin{abstract}
We completely describe the structure of the connected components of transversals to a collection of $n$ line segments in $\mathbb{R}^{3}$. Generically, the set of transversals to four segments consists of zero or two lines. We catalog the non-generic cases and show that $n \geq 3$ arbitrary line segments in $\mathbb{R}^{3}$ admit at most $n$ connected components of line transversals, and that this bound can be achieved in certain configurations when the segments are coplanar, or they all lie on a hyperboloid of one sheet. This implies a tight upper bound of $n$ on the number of geometric permutations of line segments in $\mathbb{R}^{3}$.
\end{abstract}

\section{Introduction}

A $k$-transversal to a family of convex sets in $\mathbb{R}^{d}$ is an affine subspace of dimension $k$ (e.g., a point, line, plane, or hyperplane) that intersects every member of the family. Goodman et al. [13] and Wenger [26] provide two extensive surveys of the rich subject of

\footnotetext{
* A preliminary version appeared in Proc. 15th Canad. Conf. Comput. Geom., pp. 174-177. The research of H. Brönnimann was supported by NSF CAREER Grant CCR-0133599, that of F. Sottile was supported by NSF Grant DMS-0134860, and the research of S. Whitesides was supported by NSERC and FCAR research grants.
} 
geometric transversal theory. In this paper we are interested in 1-transversals (also called line transversals, or simply transversals) to line segments. In $\mathbb{R}^{2}$ this question was studied in the 1980 s by Edelsbrunner et al. [12]: they proved that the set of transversals to $n$ line segments has total description complexity $O(n)$ and can be computed in $O(n \log n)$ time; moreover, it follows from their work that the set of transversals consists of up to $n$ connected components (see Section 3.3). Here we study the subject in $\mathbb{R}^{3}$.

We address the following basic question: What is the cardinality and geometry of the set of transversals to an arbitrary collection of $n$ line segments in $\mathbb{R}^{3}$ ? Here a segment may be open, semi-open, or closed, and it may degenerate to a point; segments may intersect or even overlap. Since a line in $\mathbb{R}^{3}$ has four degrees of freedom, it can intersect at most four lines or line segments in generic position. Conversely, it is well known that four lines or line segments in generic position admit zero or two transversals; moreover, four arbitrary lines in $\mathbb{R}^{3}$ admit zero, one, two, or infinitely many transversals [14, p. 164]. In contrast, our work shows that four arbitrary line segments admit up to four or infinitely many transversals.

Our interest in line transversals to segments in $\mathbb{R}^{3}$ is motivated by visibility problems. In computer graphics and robotics, scenes are often represented as unions of not necessarily disjoint polygonal or polyhedral objects. The objects that can be seen in a particular direction from a moving viewpoint may change when the line of sight becomes tangent to one or more objects in the scene. Since the line of sight then becomes a transversal to a subset of the edges of the polygons and polyhedra representing the scene, questions about transversals to segments arise very naturally in this context.

As an example, the visibility complex [9], [21] and its visibility skeleton [8] are data structures that encode visibility information of a scene; an edge of these structures corresponds to a set of segments lying in line transversals to some $k$ edges of the scene. Generically in $\mathbb{R}^{3}, k$ is equal to three. In degenerate configurations, however, $k$ can be arbitrarily large. Such degenerate configurations can arise, for instance, in architectural scenes, which frequently contain many coplanar edges. It is thus essential for computing these data structures to characterize and compute the transversals to $k$ segments in $\mathbb{R}^{3}$. Also, to bound the size of the visibility complex one needs to bound the number of connected components of transversals to $k$ arbitrary line segments. The present paper establishes the actual bound.

As mentioned above, in the context of three-dimensional visibility, lines tangent to objects are more relevant than transversals; lines tangent to a polygon or polyhedron along an edge happen to be transversals to this edge. (For bounds on the space of transversals to convex polyhedra in $\mathbb{R}^{3}$ see [20].) The literature related to lines tangent to objects falls into two categories. The one closest to our work deals with characterizing the degenerate configurations of curved objects with respect to tangent lines. MacDonald et al. [17] give a complete description of the set of lines tangent to four unit balls in $\mathbb{R}^{3}$. Megyesi et al. [19] describe the set of lines meeting two lines and tangent to two spheres in $\mathbb{R}^{3}$, or tangent to two quadrics in $\mathbb{P}^{3}$. Megyesi and Sottile [18] describe the set of lines meeting one line and tangent to two or three spheres in $\mathbb{R}^{3}$. A nice survey of these results can be found in [24]. Very recently, Borcea et al. [5] completed this study by characterizing the set of lines tangent to four spheres in $\mathbb{R}^{3}$.

The other category of results deals with lines tangent to $k$ among $n$ objects in $\mathbb{R}^{3}$. For polyhedral objects, de Berg et al. [3] showed an $\Omega\left(n^{3}\right)$ lower bound on the number of free (i.e., non-occluded by the interior of any object) lines tangent to four among $n$ disjoint 
homothetic convex polyhedra. Brönnimann et al. [4] showed that, under a certain general position assumption, the number of lines tangent to four among $k$ bounded disjoint convex polyhedra of total complexity $n$ is $O\left(n^{2} k^{2}\right)$. For curved objects, Devillers et al. [7] presented a simple $\Omega\left(n^{2}\right)$ lower bound on the number of free maximal segments tangent to four among $n$ unit balls, and give a bound of $\Omega\left(n^{3}\right)$ (due to Devillers and Ramos) for $n$ arbitrarily sized balls. Agarwal et al. [1] showed an upper bound of $O\left(n^{3+\varepsilon}\right)$ on the complexity of the space of line transversals to $n$ balls; recently, with Koltun, they showed that the same upper bound holds for the complexity of the set of lines that do not intersect $n$ balls [2]. Durand et al. [9] showed an upper bound of $O\left(n^{8 / 3}\right)$ on the expected number of possibly occluded lines tangent to four among $n$ uniformly distributed unit balls. Under the same model, Devillers et al. [7] recently showed a bound of $\Theta(n)$ on the expected number of maximal free line segments tangent to four among $n$ balls.

A topic closely related to line transversals is that of geometric permutations. A geometric permutation of pairwise disjoint convex objects in $\mathbb{R}^{d}$ is an ordering of the objects (or its reverse) such that the objects are met in that order by a line transversal. Worst-case bounds for general convex objects are known: $2 n-2$ is tight in two dimensions [11], while in any dimension the best known bounds are $\Omega\left(n^{d-1}\right)$ [15] and $O\left(n^{2 d-2}\right)$ [25]. The gap was closed for spheres by Smorodinsky et al. [23], who showed that $n$ spheres in $\mathbb{R}^{d}$ admit up to $\Theta\left(n^{d-1}\right)$ geometric permutations, and the same bound was also shown true for "fat" objects [16]. Recently, Cheong et al. [6] improved the known bounds for congruent balls, by showing that $n$ balls in $\mathbb{R}^{d}$ of the same radius admit at most two geometric permutations if $n \geq 9$, and at most three otherwise.

\section{Our Results}

We say that two transversals to a collection of line segments are in the same connected component if and only if one of the transversals can be continuously moved into the other while remaining a transversal in $\mathbb{R}^{3}$ to the collection of line segments. (For the sets of line transversals considered here, the notions of connected and path-connected components are equivalent since all sets are semi-algebraic.) Equivalently, the two points in line space (e.g., in Plücker space [22]) corresponding to the two transversals are in the same connected component of the set of points corresponding to all the transversals in $\mathbb{R}^{3}$ to the collection of line segments.

Our main result is the following theorem.

Theorem 1. A collection of $n \geq 3$ arbitrary line segments in $\mathbb{R}^{3}$ admits any number from zero to $n$ of connected components of line transversals. More precisely, the set of line transversals consists of at most two isolated lines unless the segments lie in one of the following three configurations:

1. the $n$ segments are all contained in lines of one ruling of (a) a hyperbolic paraboloid or (b) a hyperboloid of one sheet, or

2. they are all concurrent, or

3. they all lie in a plane, with the possible exception of a group of one or more segments that all meet that plane at the same point. 

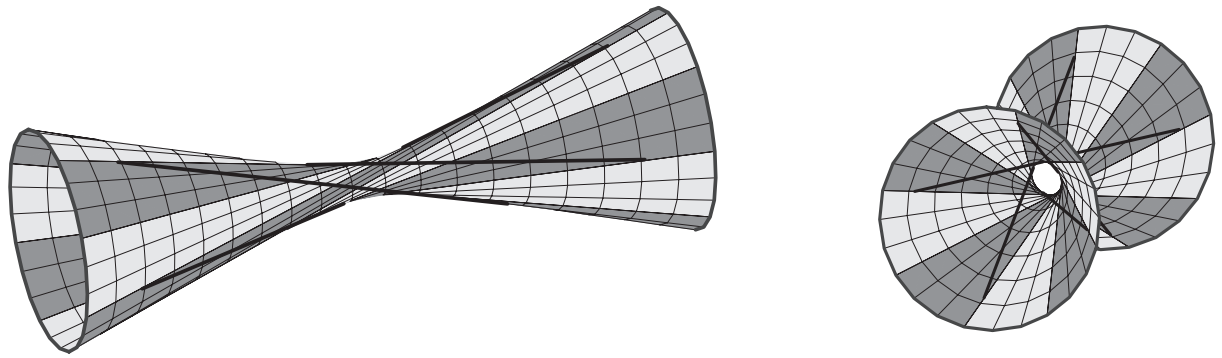

Fig. 1. Two views of a hyperboloid of one sheet containing four line segments and their four connected components of transversals (corresponding to the shaded regions). The four segments are symmetric under rotation about the axis of the hyperboloid.

In cases 1 (a) and 2 the transversals form at most one connected component. In cases 1 (b) and 3 the transversals can have any number from zero to $n$ of connected components. Moreover, in case 3 , if all segments are not coplanar, this number is at most $n-1$.

In cases 1-3 each connected component can consist of infinitely many lines or reduce to an isolated line. For example, three segments forming a triangle and a fourth segment intersecting the interior of the triangle in one point have exactly three transversals (Fig. 2(b) shows a similar example with infinitely many transversals). Also, the four segments in Fig. 1 can be shortened so that the four connected components of transversals reduce to four isolated transversals.

A simple consequence of our theorem is the following bound on the number of geometric permutations of $n$ segments in $\mathbb{R}^{3}$.

Corollary 2. A set of $n \geq 3$ pairwise disjoint segments in $\mathbb{R}^{3}$ admits up to $n$ geometric permutations and this bound is tight.

Proof. By the theorem above, $n$ segments in $\mathbb{R}^{3}$ admit up to $n$ connected components of line transversals. Within a connected component, the lines transversals must intersect the segments in the same order. Otherwise by continuity there would exist a line in that component where two objects would intersect somewhere on that line, a contradiction. Hence the upper bound. The lower bound is proved by the configuration of Fig. 1 generalized to $n$ segments: the $n$ geometric permutations are all the permutations of the form $(i, i+1, \ldots, n, 1, \ldots, i-1)$ for $1 \leq i \leq n$.

Finally, as discussed in the conclusion, an $O(n \log n)$-time algorithm for computing the transversals to $n$ segments follows directly from the proof of Theorem 1 .

\section{Proof of Theorem 1}

Every non-degenerate line segment is contained in its supporting line. We define the supporting line of a point to be the vertical line through that point. We prove Theo- 
rem 1 by considering the following three cases which cover all possibilities but are not exclusive:

1. Three supporting lines are pairwise skew.

2. Two supporting lines are coplanar.

3. All the segments are coplanar.

We can assume in what follows that the supporting lines are pairwise distinct. Indeed, if disjoint segments have the same supporting line $\ell$, then $\ell$ is the only transversal to those segments, and so the set of transversals is either empty or consists of $\ell$ and the theorem is satisfied. If some non-disjoint segments have the same supporting line, then any transversal must meet the intersection of the segments. In that case we can replace these overlapping segments by their common intersection and the theorem for the smaller collection will imply the result for the original collection.

\subsection{Three Supporting Lines Are Pairwise Skew}

Three pairwise skew lines lie on a unique doubly ruled hyperboloid, namely, a hyperbolic paraboloid or a hyperboloid of one sheet (see the discussion in Section 3 of [22]). Furthermore, they are members of one ruling, say the "first" ruling, and their transversals are the lines in the "second" ruling that are not parallel to any of the three given skew lines.

Consider first the case where there exists a fourth segment whose supporting line $\ell$ does not lie in the first ruling. Either $\ell$ is not contained in the hyperboloid or it lies in the second ruling. In both cases there are at most two transversals to the four supporting lines, which are lines of the second ruling that meet or coincide with $\ell$ [14, p. 164]. Thus there are at most two transversals to the $n$ line segments.

Now suppose that all the $n \geq 3$ supporting lines of the segments $s_{i}$ lie in the first ruling of a hyperbolic paraboloid. The lines in the second ruling can be parameterized by their intersection points with any line $r$ of the first ruling. Thus the set of lines in the second ruling that meet a segment $s_{i}$ corresponds to an interval on line $r$. Hence the set of transversals to the $n$ segments corresponds to the intersection of $n$ intervals on $r$, that is, to one interval on this line, and so the transversals form one connected component.

Consider finally the case where the $n \geq 3$ supporting lines lie in the first ruling of a hyperboloid of one sheet (see Fig. 1). The lines in the second ruling can be parameterized by points on a circle, for instance, by their intersection points with a circle lying on the hyperboloid of one sheet. Thus the set of transversals to the $n$ segments corresponds to the intersection of $n$ intervals on this circle. This intersection can have any number of connected components from zero up to $n$, and any of these connected components may consist of an isolated point on the circle. The set of transversals can thus have any number of connected components from zero up to $n$, and any of these connected components may consist of an isolated transversal. Figure 1 shows two views of a configuration with $n=4$ line segments having four connected components of transversals. 


\subsection{Two Supporting Lines Are Coplanar}

Let $\ell_{1}$ and $\ell_{2}$ be two (distinct) coplanar supporting lines in a plane $H$. First consider the case where $\ell_{1}$ and $\ell_{2}$ are parallel. Then the transversals to the $n$ segments all lie in $H$. If some segment does not intersect $H$ then there are no transversals; otherwise, we can replace each segment by its intersection with $H$ to obtain a set of coplanar segments, a configuration treated in Section 3.3.

Now suppose that $\ell_{1}$ and $\ell_{2}$ intersect at point $p$. Consider all the supporting lines not in $H$. If no such line exists then all segments are coplanar; see Section 3.3. If such lines exist and any one of them is parallel to $H$ then all transversals to the $n$ segments lie in the plane containing $p$ and that line. We can again replace each segment by its intersection with that plane to obtain a set of coplanar segments, a configuration treated in Section 3.3.

We can now assume that there exists a supporting line not in $H$. Suppose that all the supporting lines not in $H$ go through $p$. If all the segments lying in these supporting lines contain $p$ then we may replace all these segments by the point $p$ without changing the set of transversals to the $n$ segments. Then all resulting segments are coplanar, a configuration treated in Section 3.3. Now if some segment $s$ does not contain $p$ then the only possible transversal to the $n$ segments is the line containing $s$ and $p$.

We can now assume that there exists a supporting line $\ell_{3}$ intersecting $H$ in exactly one point $q$ distinct from $p$ (see Fig. 2(a)). Let $K$ be the plane containing $p$ and $\ell_{3}$. Any transversal to the lines $\ell_{1}, \ell_{2}$, and $\ell_{3}$ lies in $K$ and goes through $p$, or lies in $H$ and goes through $q$.

If there exists a segment $s$ that lies neither in $H$ nor in $K$ and goes through neither $p$ nor $q$, then there are at most two transversals to the $n$ segments, namely, at most one line in $K$ through $p$ and $s$ and at most one line in $H$ through $q$ and $s$.

We can thus assume that all segments lie in $H$ or $K$ or go through $p$ or $q$. If there exists a segment $s$ that goes through neither $p$ nor $q$, it lies in $H$ or $K$. If it lies in $H$ then all the transversals to the $n$ segments lie in $H$ (see Fig. 2(b)). Indeed, no line in $K$ through $p$ intersects $s$ except possibly the line $p q$ which also lies in $H$. We can again replace each segment by its intersection with $H$ to obtain a set of coplanar segments; see Section 3.3. The case where $s$ lies in $K$ is similar.

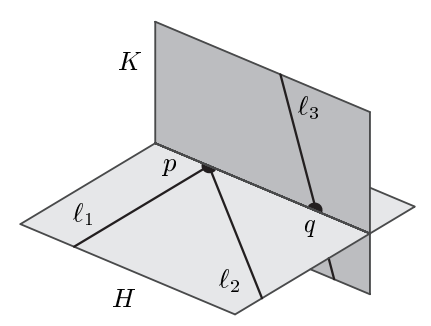

(a)

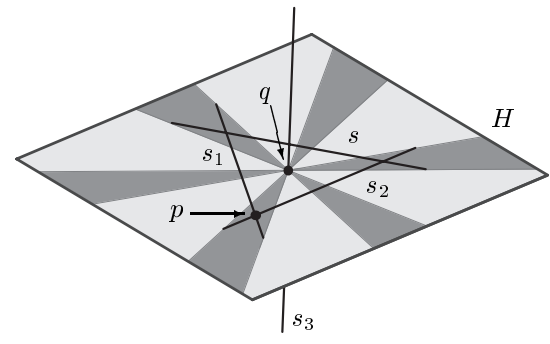

(b)

Fig. 2. (a) Lines $\ell_{1}$ and $\ell_{2}$ intersect at point $p$, and line $\ell_{3}$ intersects plane $H$ in a point $q$ distinct from $p$. (b) Four segments having three connected components of transversals. 
We can now assume that all segments go through $p$ or $q$ (or both). Let $n_{p}$ be the number of segments not containing $p$, and let $n_{q}$ be the number of segments not containing $q$. Note that $n_{p}+n_{q} \leq n$.

Among the lines in $H$ through $q$, the transversals to the $n$ segments are the transversals to the $n_{q}$ segments not containing $q$. We can replace these $n_{q}$ segments by their intersections with $H$ to obtain a set of $n_{q}$ coplanar segments in $H$. The transversals to these segments in $H$ through $q$ can form up to $n_{q}$ connected components. Indeed, the lines in $H$ through $q$ can be parameterized by a point on a circle, for instance, by their polar angle in $\mathbb{R} / \pi \mathbb{Z}$. Thus the set of lines in $H$ through $q$ and through a segment in $H$ corresponds to an interval of $\mathbb{R} / \pi \mathbb{Z}$. Hence the set of transversals to the $n_{q}$ segments corresponds to the intersection of $n_{q}$ intervals in $\mathbb{R} / \pi \mathbb{Z}$ which can have up to $n_{q}$ connected components.

Similarly, the lines in $K$ through $p$ that are transversals to the $n$ segments can form up to $n_{p}$ connected components. Note furthermore that the line $p q$ is a transversal to all segments and that the connected component of transversals that contains the line $p q$ is counted twice. Hence there are at most $n_{p}+n_{q}-1 \leq n-1$ connected components of transversals to the $n$ segments.

To see that the bound of $n-1$ connected components is reached, first consider $\lfloor n / 2\rfloor$ lines in $H$ through $p$, but not through $q$. Their transversals through $q$ are all the lines in $H$ through $q$, except for the lines that are parallel to any of the $\lfloor n / 2\rfloor$ given lines. This gives $\lfloor n / 2\rfloor$ connected components. Shrinking the $\lfloor n / 2\rfloor$ lines to sufficiently long segments still gives $\lfloor n / 2\rfloor$ connected components of transversals in $H$ through $q$. The same construction with $\lceil n / 2\rceil$ line segments in plane $K$ gives $\lceil n / 2\rceil$ connected components of transversals in $K$ through $p$. This gives $n-1$ connected components of transversals to the $n$ segments since the component containing the line $p q$ is counted twice. Figure 3(a) shows an example of four segments having three connected components of transversals.

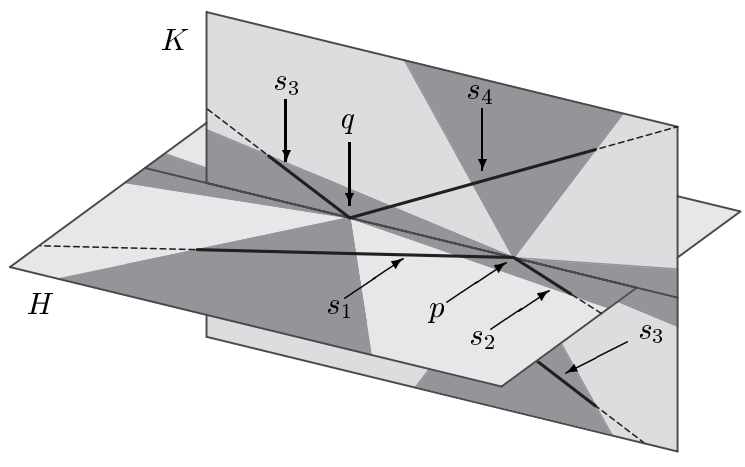

(a)

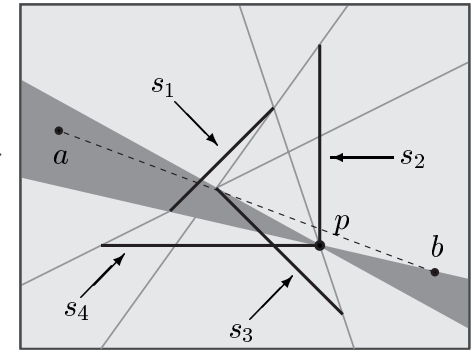

(b)

Fig. 3. (a) Four segments having three connected components of transversals. (b) Four coplanar segments having four connected components of transversals. 


\subsection{All the Segments Are Coplanar}

Let $H$ be the plane containing all the $n$ segments. There exists a transversal not in $H$ if and only if all segments are concurrent at a point $p$. In this case the transversals consist of the lines through $p$ together with the transversals lying in $H$. To see that they form only one connected component, notice that any transversal in $H$ can be translated to $p$ while remaining a transversal throughout the translation. We thus can assume in the following that all transversals lie in $H$, and we consider the problem in $\mathbb{R}^{2}$.

We consider the usual geometric transform (see, e.g., [12]) where a line in $\mathbb{R}^{2}$ with equation $y=a x+b$ is mapped to the point $(a, b)$ in the dual space. The transversals to a segment are transformed to a double wedge; the double wedge degenerates to a line when the segment is a point. The apex of the double wedge is the dual of the line containing the segment.

A transversal to the $n$ segments is represented in the dual by a point in the intersection of all the double wedges. There are at most $n+1$ connected components of such points [12] (see also Lemma 15.3 of [10]). Indeed, each double wedge consists of two wedges separated by the vertical line through the apex. The intersection of all the double wedges thus consists of at most $n+1$ convex regions whose interiors are separated by at most $n$ vertical lines.

Notice that if there are exactly $n+1$ convex regions then two of these regions are connected at infinity by the dual of some vertical line, in which case the segments have a vertical transversal. Thus the number of connected components of transversals is at most $n$.

To see that this bound is sharp consider the configuration in Fig. 3(b) of four segments having four components of transversals. Three of the components consist of isolated lines and one consists of a connected set of lines through $p$ (shaded in the figure). Observe that the line segment $a b$ meets the three isolated lines. Thus the set of transversals to the four initial segments and segment $a b$ consists of the three previously mentioned isolated transversals, the line $p b$ which is isolated, and a connected set of lines through $p$. This may be repeated for any number of additional segments, giving configurations of $n$ coplanar line segments with $n$ connected components of transversals.

\section{Algorithmic Considerations and Conclusion}

While algorithmic issues have not been the main concern of the paper, we note that the proof of Theorem 1 leads to an $O(n \log n)$-time algorithm in the real RAM model of computation. First reduce in $O(n \log n)$ time the set of segments to the case of pairwise distinct supporting lines. Choose any three of these lines. Either they are pairwise skew or two of them are coplanar. If they are pairwise skew (see Section 3.1), their transversals, and hence the transversals to all $n$ segments, lie in one ruling of a hyperboloid. Any segment that intersects the hyperboloid in at most two points admits at most two transversals that lie in that ruling. Checking whether these lines are transversals to the $n$ segments can be done in linear time. Consider now the case of a segment that lies on the hyperboloid. Its set of transversals, lying in the ruling, can be parameterized in constant time by an interval on a line or a circle depending on the type of the hyperboloid. Computing the 
transversals to the $n$ segments thus reduces in linear time to intersecting $n$ intervals on a line or on a circle, which can be done in $O(n \log n)$ time. If two supporting lines are coplanar (see Section 3.2), computing the transversals to the $n$ segments reduces in linear time to computing transversals to at most $n$ segments in one or two planes, which can be done in $O(n \log n)$ time [12].

Finally, note that we did not consider in this paper, for simplicity, segments that can extend to lines or half-lines in $\mathbb{R}^{3}$ although our theorem holds in those situations as well. For example, in $\mathbb{R}^{3}$ the transversals to $n \geq 3$ lines of one ruling of a hyperboloid of one sheet are all the lines of the other ruling with the exception of the lines parallel to the $n$ given lines. Thus, in $\mathbb{R}^{3}$ the transversals form $n$ connected components. Notice however that our theorem does not hold for lines in projective space $\mathbb{P}^{3}$; in this case our proof directly yields that if a set of lines admit infinitely many transversals, they form one connected component.

\section{Acknowledgments}

This research was initiated at the Second McGill-INRIA Workshop on Computational Geometry in Computer Graphics, February 7-14, 2003, co-organized by H. Everett, S. Lazard, and S. Whitesides, and held at the Bellairs Research Institute of McGill University. We thank the other participants of the workshop for useful discussions, and in particular Xavier Goaoc for pointing out the implication on the number of geometric permutations of segments in $\mathbb{R}^{3}$.

\section{References}

1. P. K. Agarwal, B. Aronov, and M. Sharir. Line transversals of balls and smallest enclosing cylinders in three dimensions. Discrete Comput. Geom., 21:373-388, 1999.

2. P. K. Agarwal, B. Aronov, V. Koltun, and M. Sharir. On lines avoiding unit balls in three dimensions. Proc. 20th Ann. ACM Symp. Comput. Geom., pp. 36-45, 2004.

3. M. de Berg, H. Everett, and L. J. Guibas. The union of moving polygonal pseudodiscs - Combinatorial bounds and applications. Comput. Geom.: Theory Appl., 11:69-82, 1998.

4. H. Brönnimann, O. Devillers, V. Dujmovic, H. Everett, M. Glisse, X. Goaoc, S. Lazard, H.-S. Na, and S. Whitesides. On the number of lines tangent to four convex polyhedra. Proc. 14th Canad. Conf. Comput. Geom., pp. 113-117, 2002.

5. C. Borcea, X. Goaoc, S. Lazard, and S. Petitjean. Common tangents to spheres in $\mathbb{R}^{3}$. Rapport de Recherche INRIA No. 5325, 2004. To appear in Discrete Comput. Geom.

6. O. Cheong, X. Goaoc, and H.-S. Na. Geometric permutations of disjoint unit spheres. Comput. Geom.: Theory Appl., 30:253-270, 2005.

7. O. Devillers, V. Dujmović, H. Everett, X. Goaoc, S. Lazard, H.-S. Na, and S. Petitjean. The expected number of 3D visibility events is linear. SIAM J. Comput., 32(6):1586-1620, 2003.

8. F. Durand, G. Drettakis, and C. Puech. The visibility skeleton: a powerful and efficient multi-purpose global visibility tool. Proc. 31st COMPUTER GRAPHICS Ann. Conf. Series (SIGGRAPH '97), pp. 89100, 1997.

9. F. Durand, G. Drettakis, and C. Puech. The 3D visibility complex. ACM Trans. Graphics, 21(2):176-206, 2002.

10. H. Edelsbrunner. Algorithms in Combinatorial Geometry. Springer-Verlag, Heidelberg, 1987.

11. H. Edelsbrunner and M. Sharir. The maximum number of ways to stab $n$ convex non-intersecting sets in the plane is $2 n-2$. Discrete Comput. Geom., 5:35-32, 1990. 
12. H. Edelsbrunner, H. A. Maurer, F. P. Preparata, A. L. Rosenberg, E. Welzl, and D. Wood. Stabbing line segments. BIT 22:274-281, 1982. (See also Chapter 15 of [10].)

13. J. E. Goodman, R. Pollack, and R. Wenger. Geometric transversal theory. In New Trends in Discrete and Computational Geometry (J. Pach, ed.). Springer-Verlag, Heidelberg, pp. 163-198, 1993.

14. D. Hilbert and S. Cohn-Vossen. Geometry and the Imagination. Chelsea, New York, 1952.

15. M. Katchalski, T. Lewis, and A. Liu. The different ways of stabbing disjoint convex sets. Discrete Comput. Geom., 7:197-206, 1992.

16. M. Katz and K. Varadarajan. A tight bound on the number of geometric permutations of convex fat objects in $\mathbb{R}^{d}$. Discrete Comput. Geom., 26:546-548, 2001.

17. I. G. Macdonald, J. Pach, and T. Theobald. Common tangents to four unit balls in $\mathbb{R}^{3}$. Discrete Comput. Geom., 26(1):1-17, 2001.

18. G. Megyesi and F. Sottile. The envelope of lines meeting a fixed line and tangent to two spheres. Discrete Comput. Geom., 33(4):617-644, 2005.

19. G. Megyesi, F. Sottile, and T. Theobald. Common transversals and tangents to two lines and two quadrics in $\mathbb{P}^{3}$. Discrete Comput. Geom., 30(4):543-571, 2003.

20. M. Pellegrini and P. W. Shor Finding stabbing lines in 3-space. Discrete Comput. Geom., 8:191-208, 1992.

21. M. Pocchiola and G. Vegter. The visibility complex. Internat. J. Comput. Geom. Appl., 6(3):279-308, 1996.

22. H. Pottmann and J. Wallner. Computational Line Geometry. Springer-Verlag, Berlin, 2001.

23. S. Smorodinsky, J. S. B. Mitchell, and M. Sharir. Sharp bounds on geometric permutations for pairwise disjoint balls in $\mathbb{R}^{d}$. Discrete Comput. Geom., 23:247-259, 2000.

24. T. Theobald. New algebraic methods in computational geometry. Habilitationsschrift, Fakultät fur Mathematik, Technische Universität München, Munich, 2003.

25. R. Wenger. Upper bounds on geometric permutations for convex sets. Discrete Comput. Geom., 5:27-33, 1990.

26. R. Wenger. Progress in geometric transversal theory. In Advances in Discrete and Computational Geometry (B. Chazelle, J. E. Goodman, and R. Pollack, eds.). American Mathematical Society, Providence, RI, pp. 375-393, 1998.

Received June 27, 2003, and in revised form June 30, 2004, and May 2, 2005.

Online publication August 2, 2005. 